\title{
Adverse Event following Immunization (AEFI) and COVID-19 Vaccination: A Review
}

\author{
Sharique Ahmad ${ }^{1 *}$, Shivani Singh ${ }^{1}$, Saeeda Wasim $^{2}$ and Silky Rai ${ }^{1}$ \\ ${ }^{1}$ Department of Pathology, Era's Lucknow Medical College and Hospital, Era University, \\ Lucknow, Uttar Pradesh - 226003, India \\ ${ }^{2}$ Consultant, Nova IVF Fertility, Hazratganj, Lucknow, U.P. - 226001, India
}

*Corresponding author

\section{Keywords}

Adverse event following immunization (AEFI), Vaccination, Anaphylaxis, Covid-19

Article Info

Accepted: 20 May 2021 Available Online: 10 June 2021

\section{A B S T R A C T}

Adverse event following immunization (AEFI) is basically an event of unexpected medical emergency occurring after vaccination on immunization without any causal association with vaccination. It can be any unintended, abnormal laboratory finding or anything. At the time of vaccination, there are many reasons like improper way of vaccination by health worker or quality defect of any specific vaccine. All these are the unintentional events which can lead to an adverse event in any individual. As recognition of adverse events resulted due to vaccine can be both before and after licensure. Before includes vaccination on candidate trials for evaluation of efficacy and protection. These trials mainly included various individual and can identify frequent adverse events involved after vaccination. Although, adverse events which are very rare cannot be observed during pre-licensure testing and can only be observed at the time of marketing surveillance when it come in use at wide scale. Post-marketing surveillance became complex due to the events resulting after vaccination are not mainly caused through administration of vaccine. Establishment of AEFI resulting due to any vaccination program requires careful assessment of few factors like laboratory handling, clinical factors and epidemiological factors. Its cause and effect can be enhanced by determination of rate of illness following immunization which is greater than illness in absence of vaccination. Consequently, significant attention must be required for interpreting adverse event reports associated temporarily with causality of vaccination. Currently, two complementary post-marketing system of surveillance for vaccines in United States are present. In 1978, a monitoring system was established for adverse event following immunization (AEFI) for collecting reports for public sector concerning adverse events following immunization. These reports were made from the data collected from the person who is vaccinated within 30 days of receiving vaccine. There had been some specific knowledge associated with vaccination and its adverse events like anaphylaxis associated with hepatitis B, measles thrombocytopenia and various other vaccines associated adverse events were observed which are normal but in few cases, anaphylaxis and spread of disease in immunocompromised individuals can lead to death. So, at the time of vaccination, care should be taken in assessing individuals medical history. 


\section{Introduction}

Trend of increase in vaccine curable infectious disease are known to be declining. People are very concerned towards risks involved with vaccines. Moreover, technological advancement and increased knowledge about vaccine had led to focus on vaccines present till now associated with safety which were as it now known to be the subject of concern. As AEFI is the term which helps to know these risk factors. It is inappropriate medical incidence occurring after vaccination or immunization and do not have any relationship with administration of vaccine. These incidence of adverse events could be of any non-favorable, abnormalities, symptoms or disease. If not dealt frequently and effectively can lead to undermine vaccine confidence and eventually have drastic consequence for coverage of immunization and disease prevalence. As there is nothing perfect in the world like that there is no any vaccine which can provide protection to everyone receiving it and safe too for everyone. Effective vaccines could produce few unwanted side effects mainly mild and clear up frequently. Many of the events associated with vaccine administration are literally not due to vaccine, few of them happen due to coincidental events or due to program or human error. As we all will accept it that it is impossible to speculate every person having mild to serious reaction to a vaccine. Therefore, there are some contradictions regarding few vaccines. Further, contraindications and risk of serious adverse effects can be minimized for proper functionality. Therefore, a systematic data collection, evaluation and analysis of all the minor or major medical events occurred after vaccination was ordered to perform in the immunization program by World Health Organization (WHO) (1)(2). The main aim of this immunization safety surveillance program was early observation and investigation of the events after immunization allowing the proper and instant response for AEFI emerging issues for lowering the negative impact of any individual's health and immunization program (3). Additionally, the safety surveillance program provides signal identification, generation of hypothesis, rectification and recognition of loop holes in the system for providing strength for expanding the immunization program (4). Continuous and proper observation of post-marketing vaccine, safety program data provides support for critically evaluating and communicating the updated information to public. It provided help for countering the negative approach associated with vaccination and its associated hesitancy through enhancing the clearance of immunization program (5) (6). One of the example for this had been set by surveillance program of Australia, they collect and integrate the AEFI data submitted to their respective regulator program in year 2003 (7). Although, it provided help in immunization regulation and updates of recommendations appropriately, so that, it can lead to maximize benefit of risk balance for the vaccines entered. Therefore, many expanding countries still had limited pharmacovigilance system associated with vaccine, ultimately reducing the capacity of spontaneous review of AEFI data (8).

\section{Types of events associated with AEFI}

\section{Vaccine product related reaction}

Any adverse effect can be associated with vaccine could be of many types among which product associated vaccine reaction is one. AEFI resulted from vaccine due to more than one intrinsic properties of vaccine.

\section{Vaccine quality defect-related reaction}

The adverse event resulting due to more than one defects in vaccine quality with inclusion 
of device administrated to any individual provided from manufacturer. One example of this event is failure by the manufacturer polio vaccine. An inactivated killed polio vaccine IPV must be injected for producing the desired immune response leads to the paralytic polio.

\section{Events associated with immunization error}

Adverse event could also be result of to improper handling of vaccine, prescription, administration and by their preventable nature. As seen in transmission of infection by contaminated multidose vial.

\section{Coincidental event}

The adverse event associated with immunization could also result from anxiety and their associated error. For example, fever occurring at the time of vaccination. When two or more than two events occurring around same time it is called as temporal association which shows that preceding event may or may not be related to later one. The coincidental event reflects the natural occurrence of health issues in community with common difficulties being often reported.

The immunization associated with vaccination had helped globally in eliminating small pox and poliomyelitis and many other diseases in the world. Currently, the prevention rate from immunization is estimated to be in between 23 million from various diseases like tetanus, diphtheria and measles every year of all age group. In 2011, it was reported that 83\% children were vaccinated at age 1 year of age with 3 dose of diphtheria-tetanus-pertussis (DPT3), about $84 \%$ with measles \& $88 \%$ with BCG vaccine. Immunization protection had become essential for the effectiveness of vaccine prevention and control of disease. The expectations from vaccine are greater than drugs and hurdles associated with vaccination are less tolerable for general population.
As vaccine are injected to normal healthy individual inclusion of all cohorts of infants and in huge numbers. In various places, there are particular vaccinations which are obligatory at school admission and also for International travel. The main provocation of National regulatory authorities are management, control program, licensing of medicinal products including vaccines. This had been confronted firmly by higher number of novel products and occurrence of naïve technical problems from frequent scientific advancements. Immunization advantages are frequently invisible particularly if interested disease incidence is not very high. Comparatively, incidence following immunization are very frequently seen, specifically at the time of vaccination when there had been no effects after immunization. Vaccine reaction associated alarm are found to be real or recognizable which demoralize various people from encountering vaccination. The difficulties associated with vaccine reaction and hesitation of being vaccinated has been seen for many years in industrialized countries and are frequently raised though various profits have been obtained from immunization. Immunization events is being explored in all types of countries whether high or low income, In present time, the issues arising from immunization has become very normal. The proclamation of vaccination leading to adverse incidence must be treated instantly and frequently. Defeat in doing so could result in undermining the trust or confidence in vaccine and drastic repercussion in coverage of immunization program and disease events, since very long studies proof were found about the fact that adverse events do not have any causal relationship with vaccination. Beside this, one thing should be remembered always that no any vaccine is found to be $100 \%$ safe. Error can occur due to human error or machinery error during implementation of immunization program. Therefore, adverse events associated with 
vaccine reaction and error associated with immunization incidence may lead to affect the normal healthy individual and should be quickly identified for further response. AEFI is basically unexpected medical incidence which following immunization \& does not have any cause or relationship with vaccine utilization. The adverse incidence could be any unfavorable sign, laboratory findings and symptoms of disease.

The AEFI associated events can be of many types. AEFI resulted through precipitation of vaccine due to one or more defects in quality of vaccine products. Another adverse event of immunization is found related to quality of vaccine defects associated reaction which is device administrated at the time of vaccination provided by manufacturer and quality defects more than one. Other adverse event is associated with immunization error which had been known to be caused due to improper handling of vaccine administration and prescribing. Thus, its features is preventable. One of the common event associated with vaccination is anxiety, coincidental event are also one of the adverse event caused by any other thing than vaccine product, which is called as immunization anxiety or error.

These events are assisted by causality assessment process which is a systematic review of data about an adverse event cases and also, it focuses to determine the cause in between event and vaccination. Quality of assessing the causality depends on the performance of AEFI reporting systems in context of responsibility effectiveness and investigations quality and reports. The adequate medical and laboratory services and background information approach and quality of causality review process. The causality assessment should be performed at various distinct levels. Population level is first level and it is essential to evaluate if any cause of association seen in between the vaccine usage and AEFI population. On individual level in this review, previous evidence should be done for making logical deduction for determining if any specific AEFI at individual level is found to be causally associated with vaccine usage. The last is assessment in the context of the signal investigations.

\section{Mechanism for classification of adverse events}

As vaccines are made for inducing the response through immune system which is associated with typical interaction between antigen vaccines and adjuvant which make any vaccine stronger if present, antigen presenting cells, lymphocytes and cytokine. All of this interaction is essential for formation of immunity whichever is desired against the preventable disease associated with vaccine. Though, immune response associated with vaccine is as oblivious as relatively common and mild adverse vaccine associated reaction like swelling, redness at the injection site or fever. As all the human beings have the capability of homeostasis process which helps in limiting the inflammatory response and make it short lived without any long lasting consequences. (Generally, more than one vaccine associated with immune response constituents probable not results in long term or moderate adverse events associated reaction) did not understand. It had been seen very rarely that response of immune system could lead to allergic reactions or harm to life. Vaccine product related reaction should be noticed which is very essential\& can expose the tendency for particularly greater risk individual to adverse incidence which could not be seen in most of the vaccines, like a very common inflammation seen after administration of vaccine is fever. For many of the vaccine fever is for short term and not any adverse reaction is seen associated with it. Although, a study done after vaccination program revealed that children with seizures 
disorders or toddlers and infants consisting ability of febrile seizures fever can be an activation of seizures. These cases resulted from inherent property of vaccine which causes fever. These factors in vaccine lower the seizure threshold associated with fever.

The vaccine product associated events and quality defect reaction is seen in association with route or site of vaccine administration resulting in pain during vaccination and its physiological reaction. Events associated with immune system response resulted in local effects that are usually seen at shot of injection due to various components of vaccine involving acute non-granulomatous inflammation with or without lymph node swelling as seen in diphtheria, pertussis and tetanus (DPT) vaccination which was mild to moderate. In many cases severe local inflammation were observed as swelling at injection site, pain, redness, tenderness due to intramuscular vaccine administration. Due to many components of vaccine, generalized reaction happens such as systemic inflammation (inflammation throughout in body) resulting in fever stagnation degranulation, hypersensitivity reaction or $\operatorname{IgE}$ mediated organ specific effects, autoimmune or undefined process in central nervous system like demyelinating conditions, blood \&, skin. Also after vaccination, replication associated consequences or microbial agents of vaccine and this agent could be compound present in vaccine attenuated virus or non-attenuated agent of vaccine due to deficiency at the time of vaccine manufacturing process, also a contaminant which had been introduced during manufacturing process and quality defect.

Error associated with immunization reaction explains the adverse event includes the prevention from disease. So, the classification process mainly involved error inspite of biological mechanism leading to particular
AEFI. Though various adverse events of this category results from similar process like vaccine product or quality defect involving effects. This error associated with immunization involves error in vaccine handling, resulting failure of vaccination due to inactivation of active components of vaccine, systemic or local reaction because of changes in nature of vaccine. Usage of product after its expiry leads to unsuccessful vaccination due non-viability of attenuated product of vaccine or loss of its potency.

Failure in considering appropriate warnings or precautions of vaccine use can lead to systemic immunization reactions after vaccination of wrong dose. After vaccination of incorrect product at an age group not need to be vaccinated or failure if live or attenuated product is given very early, blood components at any age can interfere with replication when antibody transferred maternally for activating the immune system.

If anyone had been injected at incorrect site it would lead to neurological, vascular, and muscular or bone injury also equipment or technique can result in any of the consequences. Error can also occur during administration of vaccine by incorrect use of diluents or product injected other than desired or intended product of vaccine leads to failure in vaccination due to properties aside from intended vaccine consist had been administrated, incorrect or inappropriate mechanism at multi-dose may result in infection at the location of shot due microbes or other contaminants developed at the site also introduced at the time of vaccination.

Failure in ensuring a safe environment during and frequently after immunization results in head injury \& syncopal episode after immunization of unintentional vaccine administration for whom not required or intentional. 
Immunization anxiety associated reactions includes vasovagal associated reaction hyperactive stress or psychiatric related disorders.

\section{Adverse events seen in post-vaccination}

Various AEFI are assigned for immunization with live attenuated vaccines as seen in the contagious disease measles, mumps, rubella (MMR) with higher mortality and morbidity which usually results in complications. MMR vaccination consist live attenuated virus which gives protection from all three disease in as single injection (9). The vaccine for MMR properly administered resulted in life time immunity in more than $85 \%$ of population? And more than $90 \%$ after two dose in population (10). The vaccine coverage at worldwide level explored due to mass vaccination and expanded program of Immunization (EPI) with significant impact on reducing this viral disease associated morbidity and also death rate (11).

This vaccine had been known to be involved in various adverse incidences. Among them minor one that noticed after vaccination includes short breathing, fever, parotitis, febrile convulsions with relatively less incidence, neurological problems. Also in many cases meningitis had been reported after 42 days of administration of mumps vaccine. (12) These events resulted in major concern for parents and health workers (13). There are various articles published for unintentional events following immunization realizing its frequency after MMR vaccination in comparison of placebo which revealed the involvement of MMR (Measles, Mumps \& Rubella) vaccine with lower grade of respiratory tract infection, irritability and similar incidence for other adverse effects were seen in comparison of placebo. As vaccination associated immunization had enabled the global elimination of small pox and poliomyelitis from western hemisphere and utmost level of reduction in incidence of other vaccine associated disease prevention in United States (14)(15). However, immunization had successfully reduced the vaccine prevention events, any vaccination can result in both types of side effects either minor or major. There should be program for public awareness about the safety associated with vaccine for reducing the controversy arose from unawareness it will lead in enhancing the coverage of vaccination. These increased vaccination coverage will elevate the adverse event associated with immunization, such events can be true reaction of vaccine or coincidental events which do not have any casual association with vaccination. Though, it will lead in clearing rumors about vaccination. Inspite of troubling for safety of vaccine, it should be safer to accept the vaccination program; it will lead to reduce the risk of disease which can be preventable through vaccination, Unless a disease had been reduced like (small pox), failure in vaccination enhances the risk in both population level and individual level. The vaccine safety response had been supported by National Child Vaccines Injury Act which revealed no fault remuneration for people get injured from vaccines (16). Also this act was made mandatory for Institute of Medicine so that they can review the scientific and nonscientific evidence related to adverse events \& significance of vaccine administrated to anyone.

Further, for reviewing all the provided information of vaccine associated adverse events after Immunization, expert committee had been established, the data mainly includes epidemiological studies, series of cases, case report of individual and testimonials of people.

The committee derives its conclusion by describing relationship in between vaccines 
and specific adverse events. They include vaccine rubella, tetanus, and hepatitis $B$ and associated disease pertussis, diphtheria and many more.

This vaccine is administrated for preventing hepatitis B which can lead to liver disease when child or any adult get infected from hepatitis B. The first shot of dose is administrated within 24 hours of birth either 2 or more dose are given after that. This is given to the person with weak immune function like HIV/AIDS and infants born premature (17)

Adverse events associated with this vaccine shown that it is safe to administrate adults and children. More than 10 million infants and children had been vaccinated with this vaccine. Its associated adverse events are normal which are normal in any vaccination. Pain at the site of injection and rise in body temperature 1-6\% reported frequently among the vaccinated people adults or children receiving vaccine (18-22). At the time of trial placebo controlled studies reveled no events or side effects, not frequently higher than among the individual receiving a placebo. Hepatitis B vaccine recommendation initiated soon after birth, which had resulted in concern about medical observation of the significantly higher number of infants after vaccination which leads in increased body temperature.

Adverse events in United States indicated an involvement among Guillain-Barré syndrome (GBS) association between first dose plasma derived hepatitis B vaccine recipients (23). However, about millions of adults received more than one dose of the recombinant vaccine of hepatitis B which do not shows any association between GBS and recombinant vaccine recipients. Reports on vaccine associated adverse events showed that approximate rate of events of anaphylaxis were found to be low in people who had been vaccinated. Only 2 case were seen of adverse events in children and among 100,763, 1 anaphylaxis case was seen in child of age group 10-11 years, New Zealand vaccine derived from plasma were administrated revealed children vaccinated with recombinant vaccine in Columbia showed no adverse event. Two event of this kind was seen in children and anaphylaxis case was seen in one among 100,763 children vaccinated with recombinant vaccine in British (D. Scheifele) and not any adverse incidence were seen among 166,757 children vaccinated with plasma derived vaccine in New Zealand. Though not any individual with anaphylaxis dies, this event could be life harming. Hepatitis B vaccine can frequently leads to life harming hypersensitivity reaction in few people. Although, vaccination of hepatitis B is contraindicated for individual vaccinated previously and had anaphylactic response for the dose of this vaccine. A wide parameter of hepatitis B immunization program was conducted among various countries for infants which revealed an Involvement between vaccination and occurrence of various moderate adverse incidence in inclusion of seizures and GBS. Any adverse event supposed to be associated with hepatitis vaccine in relation of cause and should be stabilize with risk of hepatitis B virus and its associated disease. In USA approximately 2000-5000 people in each birth cohorts died with HBV associated liver disease due to HBV infection $5 \%$ possibility. This vaccine had been introduced for infant regular vaccination. Its associated adverse incidence will be continued as an important portion of program instead of recent safety record.

Among all of this vaccine one vaccine which had been currently discovered for the wellknown viral disease COVID-19. As this disease had emerged as a pandemic so various studies had been conducted and also currently going on for its variant which are arising with greater extent now a days. Therefore, for 
establishment of any vaccine, it is very important to known any virus or bacteria structure and function, also in this viral disease its genome structure had been known it consist both structural and non-structural both types of protein, among them the spike protein plays very essential role because it had been associated with binding and fusion of virus with the cells. This leads to make it very important in vaccine formation.(24)

\section{AEFI in Covid-19 Vaccination}

COVID-19 vaccine is the intended vaccine for providing acquired immunity. Before this disease other strains of Coronavirus were seen SARS and MERS (severe acute respiratory syndrome)(Middle East respiratory syndrome). These strains helps in establishment of structural and functional knowledge of coronavirus which helps in providing the development of vaccine in early 2020 (25). Various technologies had been developed for COVID-19 are not similar to the vaccine already in use for prevention of influenza but utilizing next generation strategies associated accuracy for COVID-19 infection process $(26)(27)(28)$.

As in India had granted emergency use of authorization of two COVID-19 vaccines Covishield and Covaxin which are currently being used in the government vaccination drive. Events which are mainly seen after vaccination of covid-19 includes pain at the site of injection, headache, and myalgia and chills which are very common after any vaccination. AEFI associated with COVID -19 vaccination had been seen in at least 600 cases across the country. Vaccination perceives by the public are $10 \%$ unsafe in all vaccines, refusing the vaccination is a world wide threat in terms of heath known as vaccine hesitancy, it can lead to increase the viral spread further which can ultimately lead to the outbreak of the disease in this case COVID-19 and in other whichever vaccination program is ongoing (29). In 2020 mid, a survey was done which had reported that $60-80 \%$ of the population in the United States had accepted the vaccine against COVID-19. The difference between remaining populations not accepting the vaccination program was in education status, employment status and also the environment (30). Also a poll was conducted by National Geographic and Morning Consult which demonstrated the difference on the basis of gender for taking vaccine in the United States $69 \%$ of men were ready for vaccination in comparison of $51 \%$ women. The poll also revealed positive correlation in education and willingness of being vaccinated (31). The adverse events following immunization associated with COVID-19 vaccine are still not clear. As the effective vaccine and antiviral medicines are unavailable before (32), it is an urgent need to look and note for AEFI related to Covid-19 vaccination as due to its novelty no data is available. The mutation and protein profile analysis has revealed huge number of substitution in heterogeneous proteins of the SARSCoV-2 associated viral protein which means that response to developed vaccines will also vary.(33) Mutational divergence is important for further studies with inclusion of metabolic pathway of intraviral compounds and virus host interaction for the efficacy of vaccine post vaccination.(34)

As vaccines are widely recognized as most important and efficacious in cost interventions for health of the population, which helps in significantly reducing the morbidity and mortality of various viral or bacterial diseases. As we all want to get rid of the diseases which are not curable easily and spread with very large intensity, but on the other hand there are various kinds of hesitation from being vaccinated. AEFIs are found very rarely all the evaluations of immunization safety are recommended for all ages of individual who 
are vaccinated according the vaccine, which provides the advantage of vaccines are always found to be significantly greater than the problems they can cause and that there are not any true problems found which can lead in modifications of recommendations. Detection and quantification of mild adverse events are usually missed by passive surveillance (regular disease reporting data by all the institute). Although, conditions such as under reporting or difficulty in searching cause among adverse event and administration of vaccine which tends to hamper the pharmacovigilance. As vaccine had side effects but not as severe as disease for which any vaccine is preparing.

In conclusion, the advantages of immunization in disease prevention have significantly exceeded the risks of immunization associated adverse events. Thus for identifying vaccines associated adverse events and the features of the events revealed that we can consider vaccines as a safe biological products. Ongoing surveillance of AEFI and regular analysis and reporting data reveled integral management of immunization programs. The AEFI associated observation and reporting system should be thoroughly studied for long way enhancing the faith of the public for being vaccinated for existing or vaccine, which are newly developed for elevating the immunization on large scale in all over world.

\section{References}

1. Lim F S, Han H H, Bock H L. Safety, reactogenicity and immunogenicity of the live attenuated combined measles, mumps and rubella vaccine containing the RIT 4385 mumps strain in healthy Singaporean children. Ann Acad Med Singapore. 2007; 36: 969 - 973.

2. Makino S, Sasaki K, Nakayama T, Oka S, Urano T, Kimura M, et al., A new combined trivalent live measles (AIK-C strain), mumps (Hoshino strain), and rubella (Takahashi strain) vaccine. Am J Dis Child. 1990; 144: 905 - 910.

3. Redd S C, Markowitz L E \& Katz S L. Measles vaccine. In: Plotkin SA, Orenstein WA, Oft PA, eds. Vaccines. 3rd ed. Philadelphia: W. B. Saunders Company; 1999: 222 - 265.

4. Loo M K, Sabahi F, Soleimanjdahi H, Kazemnejad A, Roustai M H. Seroprevalence of neutralizing antibodies to measles virus in a vaccinated population in Iran, 1998. Eur J Epidemiol. 2003; 18: 1085 - 1089

5. Al-Mazrou Y, Tumsah S, Khalil M, AlJeffri M, Afzal M A, alGhamdy Y, et al., Safety evaluation of MMR vaccine during a primary school campaign in Saudi Arabia. J Trop Pediatr. 2002; 48: 354 358.

6. Jefferson $\mathrm{T}$, Price $\mathrm{D}$, Demicheli V, Bianco E. Unintended events following immunization with MMR: a systematic review. Vaccine. 2003; 21: 3954 - 3960.

7. Gooya M M, Zahraee S M, Esteghamati A R, Salaramoli M, Abdolo F, Babaee A. Expanded Program on Immunization. Health Ministry, Tehran, Iran; 2004.

8. Department of Vaccines and Biologicals WHO. Supplementary Information on Vaccine Safety: Background Rates of Adverse Events Following Immunization. Geneva: WHO; 2000.

9. CDC.Certification of poliomyelitis eradication - the Americas, 1994. MMWR 1994;43:720-2.

10. The National Childhood Vaccine Injury Act of 1986, § 2125 of the Public Health Service Act as codified at 42 U.S.C. 300aa- (suppl 1987).

11. Institute of Medicine, Howson C P, Howe C J, Fineberg H V, eds. Adverse effects of pertussis and rubella vaccines. Washington, DC: National Academy Press, 1991.

12. Institute of Medicine, Stratton K R, Howe 
C J, Johnston R B, eds. Adverse events associated with childhood vaccines: evidence bearing on causality. Washington, DC: National Academy Press, 1994.

13. Institute of Medicine, Stratton K R, Howe C J, Johnston R B, eds. DPT vaccine and chronic nervous system dysfunction: a new analysis. Washington, DC: National Academy Press, 1994

14. World Health Organization. The global eradication of smallpox: final report of the Global Commission for the Certification of Smallpox Eradication. In: History of international public health. No. 4. Geneva, Switzerland: World Health Organization, 1980.

15. CDC. Certification of poliomyelitis eradication - the Americas, 1994. MMWR 1994;43:720-2.

16. The National Childhood Vaccine Injury Act of 1986, § 2125 of the Public Health Service Act as codified at 42 U.S.C. 300aa- (suppl 1987).

17. Institute of Medicine, Stratton K R, Howe C J, Johnston R B, eds. Adverse events associated with childhood vaccines: evidence bearing on causality. Washington, DC: National Academy Press, 1994.

18. Hepatitis B immune globulin in prevention of perinatal hepatitis B virus transmission. JAMA 1987;257:2612-6.

19. Zajac B A, West D J, McAleer W J, Scolnick E M. Overview of clinical studies with hepatitis B vaccine made by recombinant DNA. J Infect 1986;13(suppl A):39-45.

20. Andre F E. Summary of safety and efficacy data on a yeast-derived hepatitis B vaccine. Am J Med 1989;87(suppl 3A):14s-20s. Vol. 45 / No. RR-12 MMWR 31

21. Szmuness W, Stevens C E, Harley E J, et al., Hepatitis B vaccine: demonstration of efficacy in a controlled clinical trial in a high-risk population in the United States. N Engl J Med 1980; 303:833-41.

22. Francis D P, Hadler S C, Thompson S E, et al., The prevention of hepatitis B with vaccine: report of the Centers for Disease Control multi-center efficacy trial among homosexual men. Ann Intern Med 1982;97:362-6.

23. Shaw F E Jr, Graham D J, Guess H A, et al., Post marketing surveillance for neurologic adverse events reported after hepatitis B vaccination: experience of the first three years. Am J Epidemiol 1988; 127:337-52.

24. Singh S, Ahmad S, Wasim S, Rai S, Gogoi S, Farheen Z. New COVID-19 Strain (SARS-Cov-2 VUI 202012/01): Lurking Challenge. Cross Current Int. J. Med. Biosci.2021;3:18-24.

25. Li Y D, Chi W Y, Su J H, Ferrall L, Hung $\mathrm{C} \mathrm{F}, \mathrm{Wu} \mathrm{T} \mathrm{C}$ Coronavirus vaccine development: from SARS and MERS to COVID-19 Journal of Biomedical Science.2020; 27(1): 104.

26. Thanh Le T, Cramer J P, Chen R, Mayhew S. Evolution of the COVID-19 vaccine development landscape. Nature Reviews Drug Discovery.2020;19:10:667-68.

27. Thanh L T, Andreadakis Z, Kumar A, Gómez R R, Tollefsen S, Saville M, et al., The COVID-19 vaccine development landscape. Nature Reviews Drug Discovery.2020;19::305-06

28. Diamond M S, Pierson $\mathrm{T}$ C. The challenges of vaccine development against a new virus during a pandemic. Cell Host and Microbe. 2020; 27: 5: 699703.

29. Dubé E, Laberge C, Guay M, Bramadat P, Roy R, Bettinger J. Vaccine hesitancy: an overview . Human Vaccines and Immunotherapeutics. 2013; 9 (8):176373.

30. Malik A A, McFadden S M, Elharake J, Omer S B. Determinants of COVID-19 
vaccine acceptance in the US. E Clinical Medicine, the Lancet. 26:2020:100495.

31. Beard, David (3 December 2020). Poll shows 61 percent of Americans likely to take COVID-19 vaccine. National Geographic. Retrieved 12 December 2020.

32. S Ahmad, T Baqar. TRALI and its Associated Risks to COVID19 19 patients Administered with Convalescent Plasma Therapy: A Review, Global Journal of Research Analysis:2020; Aug 9 (8): 4-8
33. Shivani Singh, S Ahmad, S Wasim et al., Genomic annotation of Human Coronavirus strains: A Review, Saudi J Biomed Res, Nov, 2020; 5(11): 308-319

34. S Ahmad, T Baqar, Silky Rai et al., Pathophysiological effect of novel human coronavirus (SARS-CoV-2) associated cytokine storm on vital organs: A Review, European Journal of Pharmaceutical and Medical Research, 2021, Vol 8, Issue $1: 220-225$.

\section{How to cite this article:}

Sharique Ahmad, Shivani Singh, Saeeda Wasim and Silky Rai. 2021. Adverse Event following Immunization (AEFI) and COVID-19 Vaccination: A Review. Int.J.Curr.Microbiol.App.Sci. 10(06): 555-565. doi: https://doi.org/10.20546/ijcmas.2021.1006.061 